\title{
Contexte et créativité dans les pays anglophones et francophones d'Afrique noire : essai sur le statut de l'écrivain
}

\author{
Ambroise Kom \\ (Université de Yaoundé)
}

Etudier le contexte de création en Afrique revient essentiellement à analyser la nature des rapports qui, historiquement, ont existé entre les écrivains et leurs états respectifs. Mais il s'agit moins de mesurer le niveau de ce qu'il est convenu d'appeler "engagement" de tel ou tel écrivain que de tenter de dégager le statut du créateur dans des sociétés en mutation rapide. Ainsi que le souligne Alain Ricard, en effet, l'autonomie artistique est souvent sacrifiée au nom de la prétendue construction nationale. Pourtant, écrit Ricard,

l'art doit être analysé avec des méthods particulières, il a ses propres lois, il ne faut pas plaquer des analyses socioéconomiques sur des réalités de nature artistique. [...] L'art a ses propres lois, qui ne sont pas celles de la société. Il a cependant les mêmes exigences de vérité et de liberté [je souligne]. ${ }^{1}$

L'évocation du statut de l'artiste dans l'Afrique traditionnelle peut nous permettre de mieux saisir le destin du créateur dans l'Afrique contemporaine. Dans un continent où les oeuvres d'art même les plus célèbres sont plutôt le fait d'artistes anonymes, peu d'études ont été consacrées à la personnalité de l'artiste, à ses conditions de travail et situation sociale. D'ailleurs, c'est à peine si on réussit à identifier avec précision les origines exactes de telle ou telle autre sculpture africaine. Les origines indiquées sont bien souvent approximatives tant il est vrai qu'on connaît rarement le lieu précis et la date de la production de telle ou telle oeuvre d'art. Or dans la société africaine d'aujourd'hui, toute oeuvre est généralement datée et les écrivains, y compris ceux qui se cachent derrière des pseudonymes (Ray Autra, Francesco Nditsouna, Eza Boto alias Mongo Beti, Alioum Fantouré, René Philombe, etc.) finissent toujours par être identifiés.

La fonction de 1'artiste africain traditionnel lui procure un statut social. Dans nombre de cas, l'artiste en général et le sculpteur en particulier est un spécialiste auprès de qui l'usager vient obtenir des objets dont il a besoin pour ses cultes. On retrouve là le caractère essentiellement fonctionnel de l'art africain. En plus d'être seul attitré à fabriquer des objets de culte le sculpteur 
peut être en même temps le forgeron, l'artisan chargé de fournir le village en outillage et en armements en fer (haches, couteaux, lances, poignards, etc.). Tel était le cas dans nombre de pays de l'Afrique occidentale.

Tout laisse penser qu'avec l'avènement de l'écriture, le créateur a à jamais perdu sa situation de professionnel. A cause du niveau d'instruction relativement faible partout en Afrique, le livre est loin d'être devenu un objet de consommation courante. Seuls les ouvrages didactiques, manuels scolaires et autres traités, peuvent permettre à leurs auteurs de s'assurer un revenu à peu près confortable. Et jusqu'à présent, aucun grand nom de la littérature africaine ne s'est illustré dans la préparation des ouvrages didactiques pour assurer sa survie. Faute de bénéficier d'un statut d'écrivain professionnel, les écrivains africains doivent exercer un autre métier pour gagner leur vie. Nombre d'entre eux sont enseignants, quelques-uns sont diplomates, d'autres sont employés à des degrés divers dans les administrations de leurs Etats respectifs mais l'on en trouve peu dans les professions libérales.

Dans certaines sociétés traditionnelles, l'artiste était une sorte de fonctionnaire de la cour, Jean Laude précise que :

Chez les Ba-Kuba, chacune des professions est représentée à la cour. Le représentant des sculpteurs du bois est le principal. Son insigne est une herminette au manche sculpté en forme de personnage, portée sur l'épaule gauche.

Au Bénin, le bronze était exclusivement réservé à l'Oba et à sa famille. Ceux qui le travaillaient étaient groupés en corporations (Iguneromwo) et habitaient un quartier de la ville. Les ivoiriers (groupe Igbesamwan) étaient aussi les travailleurs attitrés du bois, mais ne travaillaient pas exclusivement pour le roi. ${ }^{2}$

Les avantages du statut ainsi conféré à l'artiste ne sont évidemment pas sans conséquence sur sa production. Certains objets rituels et même usuels qu'il fabrique sont réservés à l'usage exclusif du chef. Et quand vient le moment de célébrer les hauts faits d'un règne, l'artiste doit contribuer aux manifestations par des productions de circonstance. Bien que pareilles exigences n'enlèvent pas son individualité et son style à l'artiste, il reste qu'il n'est pas libre de choisir ses thèmes et la manière de les traiter. Mais l'on doit retenir qu'il y avait une certaine harmonie entre l'artiste et sa société et singulièrement entre l'artiste et le pouvoir.

De nos jours, pareille osmose est plutôt rare. Les pouvoirs colonial et néocolonial qui ont remplacé le pouvoir traditionnel ont en général perçu l'écrivain comme un inquisiteur malveillant. Qui plus est: ces nouveaux 
pouvoirs n'étant pas l'émanation d'un vaste consensus populaire ou d'un processus démocratique, peu d'artistes dignes de ce nom ont choisi de chanter les louanges des nouvelles idéologies dominantes. Les artistes collaborateurs ou agents du système en place, ont plutôt adopté une attitude de louvoiement et se sont distingués par les ambiguïtés de leur vision du monde. Tout indique que la plupart des régimes africains contemporains se distinguent par leur mercantilisme et leur obscurantisme. La rupture entre le pouvoir et l'écrivain conscient de la fonctionalité de l'art en Afrique est ainsi devenue inéluctable. $\mathrm{Au}$ point que l'artiste qui tente de s'inscrire dans le sillage du pouvoir ressent comme une sorte de gêne, de culpabilité pour avoir pratiquement trahi un peuple dont il est censé être le porte-parole : On pourrait alors parler d'une littérature inféodée.

On connaît le rôle de l'Interprète dans la sociéte coloniale. Nanti des rudiments de la langue du maître de céans, l'Interprète était chargé de traduire le discours des administrateurs auprès des indigènes. Mais ignorant bien souvent l'idiome de ses interlocuteurs, du fait de la multiplicité des dialectes dans certains pays africains, le message n'atteignait que rarement le public visé. On peut assimiler aisément certains écrivains de la première génération à de simples interprètes.

Les intentions on ne peut plus moralisatrices d'un texte comme Les Trois volontés de Malic, ${ }^{3}$ texte visiblement commandité par le système colonial, montrent tout à fait le désir de l'auteur de célébrer les bienfaits de l'école nouvelle. Un fonctionnaire blanc y joue le rôle du bon génie. Quant à Malic, il est un héros modeste qui devient forgeron-ajusteur dans son village. Les Trois volontés de Malic est un ouvrage destiné aux enfants des colonies. Et le destin de la plupart de ces écoliers semble justement scellé dans le modèle que propose Ahmadou Mapaté Diagne. A ce titre, l'auteur peut-il être considéré comme un artiste? Outre que son mince récit à thèse est plus didactique que poétique, Diagne qui était lui-même instituteur, peut être aisément assimilé à un interprète, style nouveau.

Six ans après Les Trois volontés de Malic, il paraît sous la signature d'un autre Sénégalais, Bakary Diallo, un roman autobiographique : Force-bonté ${ }^{4}$ Analphabète jusqu'à son recrutement dans l'armée française en 1911, Bakary Diallo publie un ouvrage naïf et nostalgique de plus de deux cents pages, quelques quinze ans donc après son premier contact avec l'Europe. Aucun doute que Lucie Cousturier, sa marraine française, et ses éditeurs, lui ont été d'un énorme secours dans la mise en forme de l'ouvrage et pourquoi pas dans 
l'élaboration du message qui surgit du texte. Les récits de Diagne et de Diallo prennent en compte certaines données du contexte culturel africain du moment; il n'en demeure pas moins vrai qu'il s'agit davantage d'artistes montés par le pouvoir d'alors que d'écrivains émanant de la société africaine et de ce fait intégrés dans ladite société. L'un et l'autre ne pourront donc bénéficier que du statut que voudront bien leur reconnaître ceux-là même qui les ont fabriqués. La politique britannique d'administration indirecte n'a pas permis la naissance d'écrivains de la trempe de Diallo qui "compare les Noirs à des oiseaux nourris par une grande dame généreuse (la France) qu'ils ne cherchent qu'à aimer!"s Il n'empêche que la littérature de langue anglaise a connu dans ses rangs des évolués, individus qui, sans doute las d'attendre d'être invités dans les cercles dirigeants, ont cligné de l'oeil aux colonisateurs en montrant leur empressement à intégrer les valeurs nouvelles. Il en va ainsi de Ethiopia Unbound (1911) de l'Ethiopien Caseley-Hayford et de Eighteenpence (1943) du Ghanéen Akrofi, récits qui s'apparentent à des complaintes. L'un et l'autre auteurs s'attellent à montrer combien ils sont ouverts à la culture de l'occupant et combien ils sont prêts à se mettre à son service. L'un et 1'autre établissent un va-et-vient entre les croyances religieuses, les lois, les us et coutumes de l'univers africain et du monde européen. Ils en arrivent à reconnaître la supériorité des manières européennes. C'est le prix que Caseley-Hayford et Obeng-Akrofi paient pour mériter le statut d'artistes dans la société coloniale.

Encore que les ouvrages de l'Ethiopien et du Ghanéen comportent quelques nuances dans leur intentionalité; nuances dont ne s'embarrasse pas un Félix Couchoro (1900-1968) dans ses croisades religieuses. Ecrivain prolifique (plus de dix romans publiés), Couchoro, pour rénover le monde africain, professe l'assimilation en vantant de manière agressive les prétendus bienfaits de la morale judéo-chrétienne. Bien que Couchoro ait lutté aux côtés des nationalistes togolais dans les années 1950, il n'en demeure pas moins que l'artiste Couchoro fut un défenseur acharné de l'ordre colonial.

On le voit donc. Nombre d'écrivains africains ont poursuivi une tradition qui avait cours dans 1'Afrique précoloniale et ont agi, consciemment ou inconsciemment comme s'ils exécutaient une commande de l'Etat ou du pouvoir établi. Mais l'on sait par ailleurs que l'artiste africain traditionnel avait la responsabilité de satisfaire aux besoins de la famille et du village, c'est-à-dire du petit peuple. En littérature, il n'est évidemment pas aisé à un créateur de contenter dans son oeuvre, des groupes sociaux aux intérêts aussi divergents que l'ont été ou que le sont encore en Afrique la famille, le village et l'Etat. D'autant plus qu'ici, l'Etat n'est presque jamais l'expression de la volonté populaire. 
Nombre d'artistes ont donc eu à opérer un choix qui a marqué leur carrière et leur a conféré un statut. Si les situations de l'artiste précolonial, colonial ou postcolonial se ressemblent par certains côtés, elles sont loin d'être identiques. Procurer des objets à la famille (boîtes à bijoux, sièges, portes sculptées), au village (sculpture, statuettes, objets religieux et rituels) et commémorer les hauts faits des princes régnants n'étaient pas des actes contradictoires et ne pouvaient donc faire l'objet de conflit semblable à celui qui habiterait l'écrivain qui chercherait à produire des textes destinés en même temps aux dominants et aux dominés. Voilà qui explique l'adoption d'Ahmadou Mapaté Diagne et de Bakary Diallo par la critique coloniale et le rejet de Batouala (1921) de René Maran par la même critique. René Lebel trouve que Les Trois volontés de Malic révèle "une promesse intéressante pour l'avenir" (cité par Midiohouan, p. 61). Force-bonté est, d'après lui, "d'une lecture instructive et réconfortante" (Midiohouan, p. 67).

On ne reviendra pas ici sur le débat qui suivit la mise à l'index de Batouala. Toujours est-il que l'interdiction par l'administration coloniale d'un récit produit par un administrateur des colonies qui, on l'oublie souvent, ne rejette pas tout à fait l'idéologie coloniale, peut être considérée comme un épiphénomène. Le geste de l'administration de l'époque ne visait rien de moins qu'à promouvoir un type d'écrivain plutôt qu'un autre. Il s'agit, ainsi que le souligne Jacques Dubois, de travailler à la fabrication d'un créateur "agent du système, dans la mesure où il [l'écrivain] se place du côté d'un pouvoir et où il sera enclin à s'identifier aux forces qui assurent la conservation d'un ordre des choses".

La suite de l'histoire est connue. Quiconque produit un texte en conflit ouvert avec le système dominant tombe inexorablement sous le coup de la censure. Il en ira ainsi de Légitime défense en 1932 et du Pauvre Christ de Bomba de Mongo Beti en 1956. Mais la censure officielle ne dit pas tout. La création des Editions Présence Africaine en 1947 survient comme un défi que le monde noir entend lancer à certaines instances de production et de légitimation des oeuvres littéraires. Nombre de textes qui paraissent chez Présence Africaine énoncent des points de vue et s'adressent à une audience implicitement ou explicitement anticoloniale. Citons Coups de pillon de David Diop, Cahier d'un retour au pays natal d'Aimé Césaire et Nations nègres et culture de Cheik Anta Diop. De par ses structures et son orientation, Présence Africaine permet au monde noir de l'époque de consacrer lui-même ses artistes et de leur définir une échelle de légitimité. Pendant ce temps, l'administration indirecte semble jouer un rôle régulateur dans le domaine de l'écriture de langue anglaise. Dans ses romans, Achebe se tourne résolument vers le passé. Il affirme à ce propos : "I would be quite satisfied if my novels [...] did no more than teach their readers 
that their past--with all its imperfections--was not one long night of savagery".? L'écrivain nigérian ne recherche donc pas une consécration venue du système en place. Dans Things Fall Apart (1958), Okonkwo lutte avant tout contre les normes tribales. Il en va de même avec No Longer At Ease (1960) où Obi se trouve pris entre les réalités du groupe auquel il appartient et le mode de vie occidental qu'il a adopté. C'est avec $A$ Man of the People (1966) que se pose la problématique du rapport entre les personnages d'Achebe et la cité présente, c'est-à-dire le contexte postcolonial. Force est d'ailleurs de constater que la littérature de langue anglaise n'a connu sa véritable éclosion qu'au lendemain des indépendences. Evidemment Cyprian Ekwensi et surtout Amos Tutuola avec son Palm-Wine Drinkard (1952) pouvaient déjà être considérés comme ayant acquis une certaine notoriété. Mais il s'agit d'une consécration plutôt ambiguë tant il est vrai qu'elle ne correspond à la participation à aucun groupe ni à aucun cénacle identifiable. Ni le roman populiste d'Ekwensi ni encore moins l'oeuvre folklorique de Tutuola ne donne aux deux écrivains accès au pouvoir symbolique que détenait l'artiste dans l'Afrique traditionnelle. Les instances qui les produisent ne peuvent pas être considérées comme des instances de légitimation. Au moment où Faber et Faber publie The PalmWine Drinkard, il est difficile de dire s'il s'agit de la promotion d'une oeuvre d'art nigérianne à part ou d'un texte à fondre dans la littérature anglaise en général.

Tout se passe comme si le grand public africain anglophone n'avait pas vécu la pression coloniale avec une acuité pareille à celle des Africains de langue française. Et tout indique que même dans le domaine de l'édition, les rapports que les écrivains anglophones ont entretenus avec les diverses instances de production en Grande-Bretagne vont être rapidement régularisés. Les écrivains de langue anglaise ont assez vite bénéficié en métropole de la création des collections spécifiques dans les grandes maisons d'éditions : African Writers Series chez Heineman, Longmans African Classics, etc. En France en revanche, les

éditeurs publient indifféremment, dans les mêmes séries, auteurs français ou auteurs africains. Il en est ainsi de Julliard qui [...] publie $L$ 'Aventure ambiquë de C.H. Kane et les trois romans d'Oyono; de Plon, éditeur de Camara Laye; du Seuil, éditeur d'oeuvres d'Edouard Glissant, de Senghor, de Césaire, d'Ouologuem, de A. Kourouma, de Simone Schwarz-Bart; de Gallimard, éditeur de Black-Label de Damas et des Armes miraculeuses de Césaire; de Buchet-Chastel, éditeur de Mongo Beti, etc. ${ }^{8}$ 
Mais tout laisse penser que le statut de l'écrivain anglophone et celui de l'écrivain francophone se ressemblent depuis qu'est tombée la pluie historique des indépendances. Chez Achebe, la vision passéiste fait place à la transposition du présent. Il en va ainsi de $A$ Man of the People (1960) et de Arrow of God (1964). C'est sans doute cette prise en compte du présent qui l'a amené à interrompre sa production pendant la guerre du Biafra et lui a inspiré Girls at War après l'avoir obligé à connaître l'exil. A l'époque contemporaine, l'exil et la prison sont du reste des paramètres qui entrent en compte dans la définition du statut de l'écrivain africain. Et il ne fait aucun doute que des textes volontiers nostalgiques comme ceux de Awoonor, de Camara Laye ou des ouvrages plutôt comiques comme ceux de Sone Dipoko, de T. M. Aluko se laissent aisément éclipser par des oeuvres d'écrivains qui s'inscrivent en faux contre l'ordre social existant. En littérature africaine, c'est davantage la tradition de protestation qui triompne même si par moments certains tenors de cette tradition ont pu produire des ouvrages qui ne leur ressemblaient plus. Ainsi va-t-il de Mongo Beti avec Mission terminée ou encore de Wole Soyinka avec Aké ou les années d'enfance.

$\mathrm{Du}$ fait de sa politique d'Apartheid, la société sud-africaine est celle qui contraint le plus ses artistes à l'exil. Les créations littéraires des membres de la communauté noire s'inscrivent elles aussi en faux contre l'idéologie du groupe blanc. Toute l'oeuvre de Peter Abrahams, par exemple, traite du conflit racial; celle d'Alex La Guma en est une de violence et de protestation; celle de Mphahlele, de Denis Brutus témoignent des dures réalités de l'Apartheid, réalités qui interpellent l'artiste sud-africain comme un véritable impératif catégorique. Ici tout comme bien souvent chez l'artiste africain traditionnel, l'écrivain n'a pas le choix de ses thèmes. La communauté noire, engagée qu'elle est dans l'exploration de son passé et dans la destruction de l'image désobligeante que le Blanc donne d'elle, les lui dicte. Soyinka a d'ailleurs solennellement marqué sa fidélité à cette orientation en consacrant entièrement son discours de Stockholm de décembre 1986 à la question de l'Apartheid : lire, "Ce passé doit s'adresser à son présent" (dédié à Nelson Mandela).

Aucun doute donc que, beaucoup plus que par le passé, "le statut de l'écrivain [soit] fonction de la position occupée par cet écrivain dans le système" D'une part parce qu'aujourd'hui en Afrique, émergent des systèmes sociopolitiques nouveaux qui prennent des orientations de plus en plus précises. D'autre part, parce qu' il s'est constitué un lectorat africain qui attribue une fonction à l'écrivain en tant qu'intellectuel, "producteur actif de symboles médiatement utilisables dans le champ général des luttes idéologiques" (Dubois, p. 59). C'est dire que le statut de l'écrivain dépend ici de la position qu'il 
adopte par rapport au système dominant. Dès lors, on comprend pourquoi les rapports entre les écrivains africains et les régimes en place dans leurs pays respectifs s'organisent essentiellement en termes de conflit. Et si, comme on l'a souligné, l'Apartheid interpelle l'écrivain sud-africain, ailleurs sur le continent c'est le néocolonialisme et ses avatars qui permettent à l'écrivain contemporain de se définir. Pour illustrer mon propos, il me suffira d'évoquer les exemples de Senghor, de Ngugi wa Thiong'o, de Mongo Beti et finalement de Wole Soyinka.

Le cas Senghor est d'autant plus significatif qu'il est le promoteur d'une école littéraire; qu'il assuma pendant plus de vingt ans le pouvoir suprême dans son Sénégal natal. Tous les critiques sont d'accord : la poésie de Senghor utilise avec bonheur les ressources de la communication orale africaine. Parfaitement à l'aise dans le maniement de l'image, des métonymies, du symbole et surtout du rythme africain, Senghor se présente comme un griot moderne, comme un troubadour noir. Il s'est fait le chantre des hauts faits des héros de l'histoire africaine tels Chaka et Aynina Fall. A bien des égards, Senghor pourrait bénéficier sans conteste du statut d'artiste traditionnel. Mais son oeuvre a une portée internationale que n'atteint généralement pas l'artiste africain traditionnel.

Qui plus est : Senghor s'est trouvé mêlé aux nombreuses luttes idéologiques qui ont marqué l'évolution du monde noir au cours des cinquante dernières années. Force est pourtant de constater que l'ambiguïté de ses prises de position s'est sans cesse épaissie. Porte-parole du monde noir opprimé, Senghor a défendu et illustré avec talent les cultures africaines. Au même moment cependant, il pratiquait une politique de la main tendue au colonisateur, l'exhortant à accepter le Nègre accablé d'émotivité dans l'univers technologique qu'il fait surgir autour de lui. Ses poèmes, Ep îtres à la princesse et Prière de paix illustrent tout à fait l'appel lancé à l'adresse de l'oppresseur ou à ses semblables.

Devenu chef de 1'Etat du Sénégal en 1960, Senghor a continué de développer sa philosophie politique dans plusieurs volumes de ce qu'il a appelé Liberté. Mais le Sénégal n'est pas devenu, sous la houlette de Senghor, un pays des rêves, c'est-à-dire un espace propre à l'épanouissement intégral de l'Africain. Un contexte favorable à la créativité libre ne semble pas s'être instauré au Sénégal du fait que Senghor était à la tête de l'Etat. Certes le poète-président accueillit Camara Laye mais son geste symbolisait-il la 
connivance entre artistes conscients de la communauté de leur destin, ou étaitce un pied-de-nez à l'adresse de Sekou Touré, son terrible voisin? Les démêlés de Senghor avec Sembène Ousmane au sujet des créations de ce dernier, l'ostracisme dont Cheik Anta Diop fut victime, prouvent que Senghor, artiste au pouvoir, tenta, comme partout ailleurs en Afrique, de mettre en place un régime monocratique. Et tout indique qu'il n'hésitait pas, lui non plus, à étiqueter quiconque n'approuvait pas ses idées et sa ligne politique d'ennemi des pouvoirs publics. D'ailleurs, la répression apparaît s'être exercée au Sénégal cas de Blondin Diop, cas de Mamadou Dia - avec la même rigueur que dans beaucoup d'autres pays d'Afrique.

Pour se tenir, colloques et festivals culturels devaient nécessairement porter le sceau de l'idéologie du tenant du pouvoir, à savoir la négritude. Même les publications des Nouvelles Editions Africaines ont révélé une dépendance étroite avec les idéaux de la négritude. Tout compte fait, les institutions culturelles mises en place par le régime de Senghor (Dakar 66, colloques, NEA, etc.) se sont comportées comme de simples instances de légitimation de l'idéologie au pouvoir, c'est-à-dire de l'idéologie senghorienne. Au Sénégal d'alors, le statut d'écrivain était étroitement lié à la nature des rapports entretenus entre le producteur et la négritude gouvernante. Et quiconque avait des doutes sur le caractère inféodé de la philosophie de Senghor à l'idéologie coloniale s'est sans doute ravisé depuis que le troubadour noir s'est retiré à l'Académie Française où il occupe le siège du duc de Mirepois : Tout en se réfugiant derrière une idéologie destinée à promouvoir la culture noire, Senghor ne fut pas moins l'agent d'une superstructure néocoloniale. L'exemple du Sénégal avec Senghor, écrivain et homme politique, est révélateur des difficultés qu'ont rencontrées les artistes dans d'autres pays africains où les dirigeants étaient loin d'être des intellectuels.

Avec l'avènement des indépendances, nombre d'écrivains africains, précédemment vilipendeurs du colonialisme, marquent un temps d'arrêt dans leur élan créateur. La cible coloniale étant tombée, il a fallu attendre la mise en place d'un nouveau système pour voir comment continuer de jouer le rôle de porte-parole du petit peuple. Il en est ainsi de James Ngugi qui, authenticité oblige, se mue en Ngugi wa Thiong'o. Malheureusement, la nouvelle identité ne lui apporte pas un meilleur statut, loin s'en faut.

Lorsque Ngugi publie Petals of Blood en 1977, la critique est unanime à reconnaître la rupture entre son nouveau récit et ses trois romans précédents, Weep Not Child (1964), The River Between (1965), et A Grain of Wheat (1967). Alors que dans ses premiers écrits Nguge transposait essentiellement les luttes de l'époque coloniale, Petals of Blood est un roman prolétarien qui condamne 
sans appel le Kenya postcolonial. En présentant propriétaires et hommes politiques du Kenya comme des sangsues capitalistes, avides d'aspirer le sang des paysans et des ouvriers et en montrant que l'histoire récente du pays s'apparente à un conte macabre du fait des governants, Ngugi entend demeurer fidèle à l'option qui était la sienne pendant la période coloniale : pas de compromis avec quiconque opprime les masses populaires.

Bien plus : Ngugi crut encore mieux jouer son rôle en écrivant une pièce, en collaboration, en langue kikuyu et en la faisant jouer par des villageois au Centre Culturel et Educatif de la Communauté Kamirithu à Limuru. Après avoir attiré des foules dans un théâtre de plein air pendant plusieurs semaines, la représentation de Ngaahika Ndeenda (I will Marry When I Want) est interdite par les autorités administratives qui trouvent la pièce "provocatrice" et pas du plus grand intérêt pour la République en général. ${ }^{10}$ En effet, I Will Marry When I Want est une pièce construite autour du thème de l'exploitation économique, religieuse et sexuelle; exploitation qui aboutit à une sorte de révolte armée des masses exploitées contre les nouveaux riches, détenteurs du pouvoir.

Assez curieusement, les autorités retirent à Ngugi l'autorisation de représenter sa pièce mais ne prennent aucune mesure pour interdire l'oeuvre. on peut donc penser, suggère Lindfors, que c'est sa production en milieu rural dans une langue accessible aux masses populaires qui a le plus gêné. Et Lindfors de s'interroger :

Pourquoi la production en milieu rural de Ngaahika Ndeenda avait-elle été jugée si inopportune qu'elle avait nécessité sa suppression par le gouvernement? Après tout, la pièce par elle-même n'offrait pas une critique directe du gouvernement et n'était pas non plus impopulaire localement. En fait, si l'on en croit les comptes rendus des journaux, ce drame musical en trois heures fut un spectacle extraordinairement réussi, et l'on venait de plusieurs milles à la ronde pour le voir.

Plus que cela, c'était une entreprise communautaire, presque un modèle d'autogestion, un projet qui incluait une part hétérogène de la population de Limuru - paysans, ouvriers d'usine, petits commerçants, jeunes sans emploi, lecteurs d'université."

La suite de l'histoire est connue. Ngugi sera arrêté et persécuté. Lindfors écrit encore :

Mais la détention de Ngugi Wa Thiong'o quarante jours plus tard doit être considérée comme un acte d'une autre nature, 
d'autant plus qu'il n'y eut jamais d'accusations portées contre lui. Il semble avoir été sacrifié à la fois pour être puni à cause de ses écrits et pour servir d'avertissement à d'autres qui pourraient être tentés par son exemple, et mêleraient trop la politique à la littérature. ${ }^{12}$

$\mathrm{Au}$ bout d'un an environ d'internement, les pressions internationales feront libérer le romancier kenyan. Nais Ngugi ne sera plus autorisé à reprendre ses fonctions d'enseignant à l'université de Nairobi. Il ne pourra pas, non plus, poursuivre ses activités d'animation communautaire.

En clair, les bourgeoisies régnantes n'entendent nullement favoriser l'éclosion d'une pensée libre, surtout lorsque celle-ci ose prendre le parti de la majorité déshéritée. De manière implicite, le pouvoir invite l'artiste à participer au cénacle et à devenir agent ou acteur du système établi. Le texte de l'écrivain devrait donc, pour avoir droit de cité, s'énoncer du point de vue de ceux qui définissent les normes sociales. En somme, il faut que la pratique culturelle se conforme à l'idéologie ou à la politique du groupe dominant. Pareille exigence ne correspond ni au statut de l'artiste africain traditionnel tel qu'il nous est apparu plus haut, ni au statut de l'écrivain dans un Etat qui a des prétentions à la modernité. Il importe cependant de souligner que Ngugi wa Thiong'o n'a pas cédé aux pressions du gouvernement kenyan et qu'il affirme son indépendance en continuant de produire des ouvrages marqués au sceau de 1'anticonformisme : Devil in the Cross (1980), Detained: A Writer's Prison Diary (1981).

On le sait. En Afrique, le destin normal de tous ceux qui se singularisent à la manière de Ngugi est l'exil intérieur ou extérieur. Le cas du Camerounais Mongo Beti est connu. Bien que l'exil extérieur lui ait jusqu'ici épargné la prison, le cheminement artistique de Mongo Beti s'apparente à celui de Ngugi, même type de combat pendant la colonisation, long silence dès l'avènement de l'indépendance et retour à l'écriture pour dénoncer les compromissions de toutes sortes qui contraignent l'Afrique à tourner en rond. Mais Beti étant personnellement hors d'atteinte, les oligarchies gouvernantes s'attellent à mettre ses écrits à l'index pour empêcher le public d'y avoir accès. Les techniques adoptées sont telles qu'on ne sait jamais lequel des ouvrages de Beti est véritablement interdit. Certains arrêtés d'interdiction, pudeur oblige, ne font l'objet d'aucune publicité et même d'aucune publication. Pourtant, les descentes de police dans les librairies et les saisies en douane sont en général des indices qui ne trompent pas. Or, la plupart des ouvrages de Mongo Beti, depuis Main basse sur le Cameroun (1972) jusqu'à sa revue Peuples noirs, 
peuples africains en passant par Perpétue et l'habitude du malheur (1974) ont été censurés d'une manière ou d'une autre.

Mongo Beti n'est pas le seul à avoir été ainsi traité au Cameroun. René Philombe, malgré sa paralysie, fut persécuté et jeté en prison à cause de ses écrits et de son statut de libre penseur. Bernard Nanga fut interdit de parole à la suite de la publication des Chauves-souris (1980). A l'université de Yaoundé où il enseignait la philosophie, le chancelier de l'époque prit un texte pour exiger de tout conférencier de lui "remettre le texte de sa conférence au moins une semaine avant la date prévue pour ladite conférence" (circulaire no 160/UY/SG du 25 mai 1981). Il s'agissait là d'une forme insidieuse de censure mise en place pour empêcher Bernard Nanga de faire la moindre déclaration publique sur son récit par ailleurs introuvable dans les librairies de la ville à l'époque.

On comprend dès lors que les batailles menées et souvent gagnées par Wole Soyinka soient perçues comme un véritable combat d'avant-garde dans le contexte postcolonial. Même s'il a commencé à produire avant les années soixante, c'est bien avec l'avènement des indépendances que se confirme la notoriété de l'écrivain nigérian, notoriété fondée sur une oeuvre tournée vers l'avenir mais qui s'enracine dans les profondeurs de l'Afrique millénaire. Totalement versé dans les mythes et les rituels yoruba, Soyinka a créé des tragédies qui, pour être proches de la tragédie grecque, restent on ne peut plus fidèles à l'esprit d'Ogun, le Dieu Yoruba de la créativité. Héritier incontestable de la tradition théâtrale yoruba, Soyinka se sert du genre à la manière de ses ancêtres, c'est-à-dire pour faire passer la satire de la société. Soyinka se distingue aussi par ses croisades contre certains régimes qui ont gouverné le Nigéria.

Réfractaire aux doctrines et aux idéologies d'où qu'elles viennent, Soyinka, au-delà de son style ésotérique, est connu pour son franc-parler et son indépendance d'esprit. Défenseur inlassable de la personnalité africaine, Soyinka est un artiste qui ne transige pas avec la liberté de dire et de faire. Malgré les coups de semonce reçus, il se veut le porte-parole du petit peuple et tourne systématiquement le dos à l'élite qu'il traite dans The Man Died (écrits de prison) de "couches d'esclaves privilégiés qui sont les fondations des palais de marbre des tyrans de l'heure". Soyinka lutte pour l'avènement du changement auquel il croit. Voilà qui explique pourquoi il s'en prend avec une violence de langage peu commune aux dirigeants corrompus qui dilapident impunément les fonds publics dans des projets sans lendemain (FESTAC, GREEN REVOLUTION, ABUJA, etc.). Soyinka n'hésite pas non plus à créer des pièces de circonstance pour s'attaquer à certains fléaux sociaux tels que le 
charlatanisme, la futurologie ou le fanatisme religieux qui freinent l'évolution de son pays et du continent.

Un peu partout en Afrique, les différents régimes usent de tous les prétextes pour lutter contre l'émergence d'une pensée libre. On comprend de ce fait le symbole que représente la consécration de Soyinka. Senghor nous avait appris à chanter, à pleurer, à prier et à pardonner. Ailleurs en Afrique, on est heureux lorsqu'on ne dit jamais rien, ne voit jamais rien et $n$ 'entend jamais rien. Même la plupart des institutions éducatives et culturelles (écoles, universités, maisons d'éditions, etc.) préparent de simples griots tant les systèmes s'acharnent à exiger de la gratitude pour l'hypothétique générosité des dirigeants.

Tout se passe en définitive comme si c'est à l'Etat que l'artiste africain d'aujourd'hui et de demain devra son statut, au risque d'être traité de déviant, de subversif, c'est-à-dire de traître à la nation. Rien n'est donc épargné pour faire en sorte que l'artiste, produit du système établi, devienne un simple agent dudit système. Assez paradoxalement, le succès remporté par Soyinka a été applaudi par les responsables politiques africains au plus haut niveau : chefs d'Etat, ministres, etc. Est-ce à dire que ces derniers ont compris que 1'attribution du Prix Nobel 1986 de littérature à Wole Soyinka consacrait l'échec de leur tentative de dompter et d'apprivoiser l'écrivain africain contemporain? On pourrait alors penser que dorénavant, les responsables des instances culturelles et politiques des pays d'Afrique laisseront à l'artiste le loisir de se forger un statut. L'exemple de Soyinka le confirme : cesser de flatter les princes régnants et même prendre le risque d'aller à contre-courant de l'idéologie officielle n'est pas nécessairement antipatriotique, mais fait partie de la contribution de l'artiste à l'élaboration d'une conscience et d'une culture nationales. Car, écrit Soyinka,

... quand l'écrivain ne peut plus faire fonction de conscience au sein de sa société, il doit admettre qu'il n'a plus que deux choix devant lui : ou bien se renier totalement ou bien se replier sur une position de chroniqueur et de médecin légiste. Mais il ne peut pas continuer à se distraire en se préoccupant de problèmes universels. Ceux-ci répandent du baume sur les blessures abstraites mais ils ne soignent pas les plaies béantes de l'humanité noire. C'est en se préoccupant de culture, non de mythologie, que l'on consolide une société africaine, la fonction de l'artiste a toujours été d'enregistrer les moeurs, les usages de sa société $e t$, en même temps, de faire entendre la 
voix visionnaire de son époque. Il est grand temps que l'écrivain retrouve ce rôle fondamental qui est le sien. ${ }^{13}$

Reste donc à espérer que les Etats africains ne tarderont plus à faire les concessions qui s'imposent pour permettre à leurs fils et à leurs filles de contribuer à leur forger en toute quiétude, une identité culturelle pouvant s'imposer partout et en tout temps comme valeur de référence.

\section{NOTES}

1 Notre Librairie, no. 83 (Avril-Juin 1986), pp. 96-97.

2 Jean Laude, Les Arts en Afrique noire (Paris : Le Livre de Poche, 1966), p. 129.

${ }^{3}$ Les Trois volontés de Malic (Paris : Larose, 1920).

${ }^{4}$ Force-bonté (Paris : Rieder et Cie, 1926).

5 Guy Ossito Midiohouan, L'Idéologie dans la littérature négro-africaine d'expression française (Paris : 1'Harmattan, 1986), p. 67.

6 Jacques Dubois, L'Institution de la littérature (Paris/Bruxelles: Nathan/ Labor, 1983), p. 106.

7 Cité par O.R. Dathorne, The Black Mind (Minneapolis : University of Minnesota Press, 1974), p. 157.

8 B. Mouralis, Littérature et développement (Paris : Silex, 1984), p. 149.

9 Dubois, op. cit., p. 103.

${ }^{10}$ Lire à ce propos l'article de Bernth Lindfors, "La détention de Ngugi Wa Thiong'q", dans Peuples noirs, peuples africains, no. 26 (Mars-Avril, 1982), pp. 73-88.

11 Ibid., p. 79. 
12 Ibid., p. 81.

${ }^{13}$ Cité dans Anthologie critique de la littérature africaine anglophone (Paris : Union générale d'éditions 10/18, 1983), p. 169.

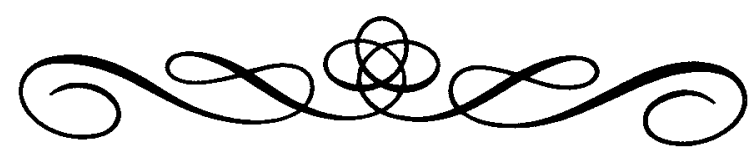

